Ingeniare. Revista chilena de ingeniería, vol. 17 N³, 2009, pp. 337-346

\title{
RESTAURACIÓN DE SERVICIO MULTIOBJETIVO EN REDES DE DISTRIBUCIÓN UTILIZANDO NSGA-II
}

\section{MULTIOBJECTIVE SERVICE RESTORATION IN POWER DISTRIBUTION SYSTEMS USING NSGA-II}

\author{
Jorge Mendoza Baeza ${ }^{1} \quad$ Fabian Rojas Lago ${ }^{1}$ \\ Recibido 3 de Octubre de 2008, aceptado 30 de junio de 2009 \\ Received: October 3, 2008 Accepted: June 30, 2009
}

\begin{abstract}
RESUMEN
Este trabajo presenta un modelo que permite resolver el problema de la restauración del servicio en las redes eléctricas de distribución de media tensión. El algoritmo fue desarrollado en ambiente MATLAB y es capaz de encontrar aquel conjunto de topologías que minimizan dos objetivos en forma simultánea bajo el concepto de dominancia de Pareto. La búsqueda de estas soluciones eficientes se logra utilizando una técnica evolutiva de optimización multiobjetivo denominada NSGA-II, la cual es combinada con una poderosa estrategia de codificación de individuos y operadores especialmente desarrollados para los problemas de configuración de redes eléctricas. Los principales resultados de este trabajo se centran en la capacidad del algoritmo para encontrar el conjunto de soluciones eficientes y el reducido tiempo de simulación requerido. Esta herramienta permite que los despachadores del sistema puedan tomar rápidas decisiones, incluso bajo condiciones de múltiples fallas en la red.
\end{abstract}

Palabras clave: Restauración de servicio, sistemas de distribución, multiobjetivo.

\begin{abstract}
A model to solve the service restoration problem in medium voltage distribution networks is developed in this work. The algorithm was developed in MATLAB and is able to find a set of topologies that minimize two objectives under the concept of Pareto dominance. The search of efficient solutions is attained using the evolutive multiobjective optimization technique called NSGA-II together with a powerful codification strategy and genetic operators specially developed for the configuration of the electric network. The main results of this work focused on the ability of the algorithm to find the set of efficient solutions and reduced simulation time. This tool enables system dispatchers to make quick decisions, even under conditions of multiple failures in the network.
\end{abstract}

Keywords: Service restoration, distribution system, multiobjective.

\section{INTRODUCCIÓN}

Los sistemas eléctricos de distribución (SD) de media tensión normalmente son redes que funcionan de manera radial. Esta configuración permite un diseño y operación de las protecciones de manera más simple y confiable. No obstante, la desventaja de esta estructura es que ante una falla del sistema gran parte de la red podría quedar sin suministro eléctrico. Es por esto que generalmente los SD se diseñan de tal forma que las cargas puedan ser alimentadas a través de más de una alternativa.
En aquellos casos en que ocurre una falla temporal, son los equipos reconectadores los encargados de restaurar el servicio [1]. No obstante, ante una falla permanente, una serie de acciones deben ocurrir de tal forma de permitir la restauración del sistema. En primer lugar, la falla debe ser detectada y posteriormente localizada. Luego de esto, ella debe ser aislada a través de los elementos de maniobra (EM) más cercanos al punto de falla. Luego, es necesario llevar a cabo un proceso por medio del cual se restaure el servicio a la mayor cantidad de usuarios y en el menor tiempo posible.

\footnotetext{
1 Escuela de Ingeniería Eléctrica. Pontificia Universidad Católica de Valparaíso. Casilla 4059. Valparaíso, Chile. E-mail: jorge.mendoza@ucv.cl; fabian1770@gmail.com
} 
Lo anterior debe ser realizado manteniendo la estructura radial de funcionamiento, considerando la capacidad de reserva de los alimentadores y las restricciones propias del funcionamiento de una red eléctrica. Por consiguiente, la restauración puede ser considerada como un problema de asignación, donde se plantea la transferencia de cargas del área afectada a alimentadores adyacentes por medio de interruptores fronterizos.

Debido a que hoy en día los sistemas eléctricos son cada vez de mayores envergaduras y más complejos, esta toma de decisión se hace muy difícil de realizar sin una herramienta de optimización que sirva de apoyo.

Por lo tanto, la restauración de servicio por definición es un problema de optimización combinatorial, no-lineal y sujeto a restricciones de operación de la red. Diversas técnicas y enfoques al problema de la restauración son expuestos en [2], mostrando una serie de artículos desarrollados entre los años 1981 y 2001. En este estado del arte, se presentan principalmente métodos que resuelven el problema utilizando sistemas expertos y métodos heurísticos.

En este sentido, históricamente la restauración del servicio en redes eléctricas ha sido entendida como un problema con múltiples objetivos, no obstante éstos siempre han sido abordados de manera monoobjetivo utilizando sistemas expertos [3-5] y reglas heurísticas [6-8]. Entre los objetivos considerados en estos trabajos están el reducir el número de clientes sin servicio, número de operaciones de los elementos de maniobra, desviaciones de voltaje, corrientes de línea, pérdidas, márgenes de potencia, desbalance en los alimentadores e índices de confiabilidad [9].

Por otra parte, en el problema de la restauración también han sido utilizadas otras técnicas de optimización basadas en la inteligencia artificial; en el trabajo presentado en [10], se comparan 4 técnicas heurísticas modernas, entre ellas, Búsqueda Tabú, Búsqueda Tabú Reactiva, Simulación Templada Paralela y Algoritmos Géticos.

En los últimos años se han estado uniendo varias técnicas con el objetivo de alcanzar mejores soluciones. En [11] se mezclan los Algoritmos Genéticos con Búsqueda Tabú y en [11] se unen la Lógica Difusa y el método de Grey.

No obstante, los importantes avances en estos trabajos, existe un reducido número de artículos que han logrado abordar el problema de la restauración de servicio a través de verdaderas técnicas de optimización multiobjetivo. Esta clase de técnicas está orientada a encontrar un conjunto de soluciones compromiso o eficientes entre los objetivos considerados. La idea principal es entregar al agente encargado de la toma de decisiones, un conjunto reducido de soluciones, que minimicen de forma simultánea los objetivos.

El trabajo presentado en [13] muestra un modelo que permite encontrar las soluciones en compromiso entre las pérdidas de potencia y el margen de potencia de los alimentadores. Allí se utiliza el método Non-dominated Sorting Genetic Algorithm (NSGA) y de Fuzzy Evolutionary Strategy (FES), en un método compuesto denominado FES-NSGA. En [14] se utiliza NSGA de segunda generación (NSGA-II) para encontrar las soluciones de compromiso entre el índice de cargas sin suministro, las pérdidas y el número de operaciones de los elementos de maniobra. Este artículo detalla muy bien el algoritmo propuesto y compara sus resultados con una propuesta basada en NSGA de primera generación, mostrando las ventajas del uso de esta técnica. Sin embargo, este artículo utiliza una codificación binaria que, como se demuestra en [15], tiene desventajas respecto a una codificación de los individuos utilizando números reales en este tipo de aplicaciones.

Considerando las investigaciones realizadas en esta área, y debido a la importancia que tiene para los sistemas de distribución la oportuna restauración del servicio eléctrico, el objetivo de este artículo es mostrar el desarrollo de una herramienta que permita la asistencia en la toma de decisiones de los despachadores en los sistemas de distribución.

Por esta razón, considerando los importantes resultados arrojados en [13-15], este trabajo aborda el problema de la restauración del servicio utilizando NSGA-II como algoritmo de búsqueda de las soluciones eficientes, destacando como principal diferencia respecto de [14], la utilización de una codificación mejor adaptada al problema de configuración de las redes eléctricas.

La mayor contribución de este artículo puede ser resumida de la siguiente manera:

1) Utiliza una técnica de optimización multiobjetivo con ventajas probadas sobre otros métodos.

2) Incluye una codificación y operadores genéticos que permiten una mejor exploración del universo de posibles soluciones, utilizando menores tiempos de simulación.

3) Es capaz de restaurar el sistema ante condiciones de múltiples fallas.

4) Está desarrollado para utilizar diferentes objetivos, dando flexibilidad en la búsqueda de las configuraciones. 


\section{FORMULACIÓN DEL PROBLEMA}

Diversas funciones objetivo han sido utilizadas en el problema de la restauración del servicio, las cuales pueden ser clasificadas según las siguientes categorías y que corresponden a la minimización de [2-14]:

- Variables técnicas: Desviación de tensión, corrientes por las líneas, carga de los trasformadores.

- Asociados a la confiabilidad: Duración media de Interrupciones del sistema (SAIDI), energía no suministrada.

- Asociados al tiempo: Tiempo de restauración, número de maniobras.

- Asociados a la carga: Número de clientes sin servicio, potencias sin servicio.

- Asociados a las pérdidas: Reducción de pérdidas.

Bajo la perspectiva del problema de la restauración, de estas 5 categorías podemos indicar lo siguiente. La primera, asociada a las variables técnicas, no necesariamente requiere de minimización, sino que sólo asegura que los límites no sean sobrepasados. La segunda, asociada a indicadores de confiabilidad, no es indicadora representativa para optimizar una red que se encuentra en una etapa de transición, ya que por definición éstos son indicadores probabilísticos de una confiabilidad futura.

Tanto los objetivos de minimización de los tiempos de restauración, asociados al número de maniobras (NM) como la disminución de las cargas sin servicio, son los aspectos más relevantes ante el estado de contingencia del sistema. Aunque la minimización de pérdidas $(\mathrm{P})$ podría considerarse como un objetivo secundario, hoy en día existe una constante preocupación en el uso eficiente de la energía. Por esta razón este trabajo centra sus objetivos de optimización en maximizar la mayor cantidad de cargas con servicio, disminuir el número de maniobras para restablecer el servicio y la reducción de pérdidas.

En el caso de la maximización de la mayor cantidad de cargas con servicio, esto se logra aislando la falla a través de aquellos EM más cercanos a la falla. Los otros dos objetivos son parte del proceso de optimización propuesto según las ecuaciones (1) y (2).

$$
\begin{gathered}
\operatorname{Min} P(x)=\operatorname{Pérdidas}(x) \\
\operatorname{Min} N M(x)=\sum_{i=1}^{s}\left|s w_{i}-x_{i}\right|
\end{gathered}
$$

Las restricciones consideradas en este problema son: a) Estructura radial

b) Voltajes dentro límites permitidos

c) $v_{\min }<v_{i}<v_{\max }$

d) Corrientes dentro límites permitidos

$$
i_{i}<i_{\max }
$$

En esta nomenclatura $x$ representa la topología de la red, $s$ es el número de EM de la red, $s w_{i}$ el estado del $i$-ésimo EM justo antes de la falla y $x_{i}$ el estado del $i$-ésimo EM con la nueva topología. Por otra parte la $i_{i}$ es la corriente en la $i$-esima línea e $i_{\max }$ el máximo valor de corriente permisible.

\section{ALGORITMO DE OPTIMIZACIÓN MULTIOBJETIVO (NSGA-II)}

El NSGA-II es un algoritmo de optimización multiobjetivo basado en los métodos de programación evolutiva. En particular NSGA-II es un algoritmo de segunda generación propuesto por Deb el año 2001 [15], siendo una mejora al algoritmo original propuesto por Srinivas y Deb.

El objetivo de estos algoritmos es encontrar un conjunto de soluciones eficientes bajo el concepto de dominancia de Pareto. La definición de dominancia de Pareto (asumiendo minimización) para dos vectores de decisión $\boldsymbol{x}, \boldsymbol{y} \in \mathrm{F}$ ( $F$ se refiere a la región factible), indica lo siguiente:

Un vector $\boldsymbol{x}=\left(x_{1}, x_{2} \ldots x_{k}\right)$ se dice que domina (en el sentido de Pareto) a otro vector $\boldsymbol{y}=\left(y_{1}, y_{2} \ldots y_{k}\right)$ (que se denota como $(\boldsymbol{x} \prec \boldsymbol{y})$ sí y solo si:

$$
\forall i \in(1, \ldots, k), x_{i} \leq y_{i} \wedge \exists i \in(1, \ldots, k): x_{i}<y_{i}
$$

En otras palabras, un vector domina a otro, cuando éste es menor o igual para todos los componentes y es estrictamente menor en al menos uno de ellos. Es importante notar que si una solución $\boldsymbol{x}$ no domina a otra solución $\boldsymbol{y}$, e $\boldsymbol{y}$ no domina a $\boldsymbol{x}$, entonces ambos son no-dominados, es decir, no pueden ser comparados.

Las principales etapas del NSGA-II se describen a continuación:

1) Generar una población aleatoria $P$ de tamaño $N$.

2) Identificar los frentes de dominancia: Para esto primero, por cada solución, se calculan 2 valores: (i) $n_{i}$, el número de soluciones que dominan a la 
solución $i$, y (ii) $S_{i}$, un conjunto de soluciones las cuales la solución $i$ domina. Posteriormente se identifican todos los puntos que tienen $n_{i}=0 \mathrm{y}$ se almacenan en una lista $F_{l}$. Se llama $F_{l}$ a la frontera actual. Ahora, para cada solución en la frontera actual se visita cada miembro $(j)$ en su conjunto $S_{i}$ y reduce su $n_{j}$ uno a uno. De esta forma, si para cualquier miembro de $j$ el valor se vuelve cero, se pone en una lista separada $H$. Cuando todos los miembros de la frontera actual han sido chequeados, se declara a los miembros de la lista $F_{1}$ como miembros de la primera frontera. Luego se continúa el proceso usando la nueva frontera $H$ como la frontera actual.

3) Para mantener la diversidad en la frontera de Pareto, se calculan las distancias de apilamiento en cada frente. Para esto se debe calcular el perímetro del cuboide formado por las soluciones vecinas que poseen el mismo rango de dominancia que la alternativa $i$, lo cual se describe por medio de la siguiente ecuación.

$$
d i=\sum_{m=1}^{M}\left|\frac{f m^{I_{i+1}^{m}}-f m^{I_{i-1}^{m}}}{f m^{\max }-f m^{\min }}\right|
$$

Donde $I^{m}$ es un vector que indica la alternativa de solución vecina a la alternativa $i, \mathrm{fm}^{\max }$ y $\mathrm{fm}^{\mathrm{min}}$ son los valores máximos y mínimos sobre todo el espacio de soluciones de la función objetivo $m$, y $M$ es el número de funciones objetivos optimizadas.

4) Usando selección, cruzamiento y mutación se genera una población descendiente del mismo tamaño $P$.

5) Reunir padres e hijos en un conjunto de tamaño $2 N$, clasificar los frentes de dominancia y distancias de apilamiento.

6) Determinar el conjunto descendiente final seleccionando los frentes de mejor rango. Si se supera el límite de población $N$, eliminar las soluciones con menor distancia de apilamiento en el último frente seleccionado.

7) Si se cumple el criterio de convergencia, se finaliza el proceso. De lo contrario retornar al paso 4.

\section{CODIFICACIÓN Y OPERADORES GENÉTICOS}

En este artículo se utilizó una estrategia de codificación de los individuos (topologías) desarrollada para los problemas de reconfiguración [17], la cual tiene una gran adaptabilidad debido a la manera en que explora el universo de las posibles soluciones comparativamente con otras técnicas [18] y que además ha sido aplicado al problema de reconfiguración multiobjetivo [19]. Sin embargo, en este trabajo se demuestra que tiene una mejor adaptabilidad al problema de restauración de servicio.

Este tipo de codificación está basado en un vector de números reales que identifican aquellos elementos de maniobra que se encuentran presentes en líneas asociadas a las mallas fundamentales del sistema. Bajo esta estrategia se define una malla fundamental, como aquella malla que puede ser formada en el sistema y que no contiene a otras mallas.

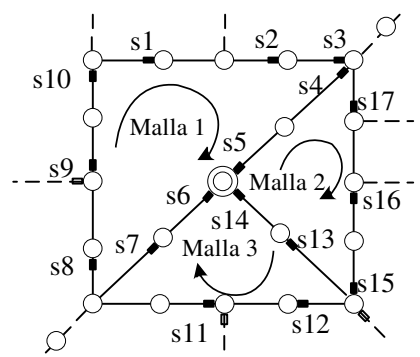

Figura 1. Red de distribución enmallada.

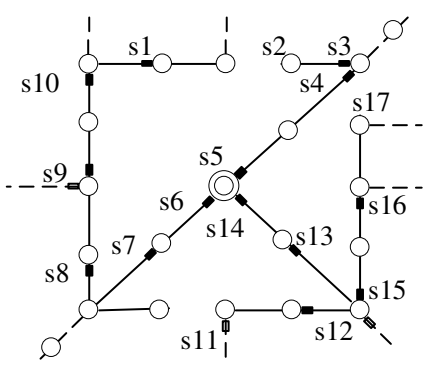

Figura 2. Red de distribución radializada.

En el sistema de la Figura 1, al cerrar todos los elementos de maniobra, es posible formar 3 mallas fundamentales. Estas mallas están constituidas por los elementos mostrados en (5). Si se escoge un elemento de cada malla, que no haya sido seleccionado previamente, es posible formar fácilmente topologías radiales; por ejemplo, la topología de la Figura 2 se obtiene si se escogen los elementos [2 17 11] respectivamente. Sin embargo, existe una pequeña probabilidad que al seleccionar ciertos elementos de la red, algunas configuraciones no presenten conectividad; es por esta razón que se utiliza un filtro que permite descartar aquellas configuraciones que no cumplen dicha condición. Esta rutina es muy rápida y simple, ya que sólo necesita verificar conectividad de los nodos del sistema. Una comparación de esta metodología respecto de otras alternativas se detalla en [17]. 


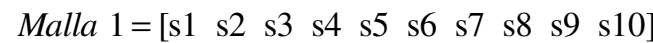

Malla $2=\left[\begin{array}{llllll}\text { s4 } 4 \text { s5 } & \text { s13 } & \text { s14 } & \text { s15 } & \text { s16 } & \text { s17 }\end{array}\right]$

Malla $3=\left[\begin{array}{llllll}\text { s6 } & \text { s7 } & \text { s11 } & \text { s12 } & \text { s13 } & \text { s14 }\end{array}\right]$

Una de las principales ventajas de esta codificación es que no todos los EM son considerados como elementos candidatos a la apertura, sino que solo aquellos que están asociados a las mallas fundamentales del sistema, descartándose aquellos que se encuentran en zonas sin abastecimiento alternativo. Otra de las grandes ventajas es que una codificación en números reales permite disminuir drásticamente la cadena (string) representativa de la topología, respecto de las codificaciones binarias. En el caso de Figura 1, el sistema podría tener muchas líneas, barras y elementos de maniobra, no obstante al tener 3 mallas el string representativo de una topología radial solo tendrá 3 elementos. Además, los vectores de mallas fundamentales son usados para guiar a los operadores de cruza y mutación. Respecto de los operadores genéticos, el NSGA-II utiliza tanto la mutación como la cruza, siendo esta última el operador principal. Bajo la codificación utilizada es posible aplicar los operadores desarrollados en [17], los cuales son ejemplificados en la Figura 3. Para mayor detalle sobre la codificación y los operadores aplicados pueden ser vistos en la referencia respectiva.
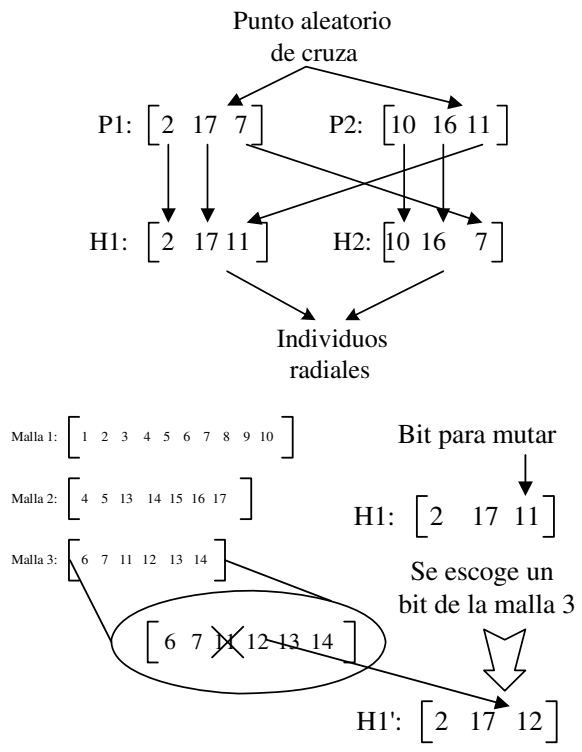

Figura 3. Ejemplo de la aplicación de la cruza y mutación.

La aplicación de esta codificación al proceso de restauración conlleva a algunas modificaciones. Considere que actualmente la red de la Figura 1 se encuentra operando con la apertura de los EM [2 17 11], (Figura 2).
Ocurre una falla permanente que es aislada con la apertura de los EM s9 y s10. Debido a esta condición de emergencia, las tres mallas fundamentales de este sistema (5) quedan reducidas solo a dos, al desaparecer la malla 1 (6).

Lo anterior permite simplificar el problema, incluso el considerar eventuales fallas múltiples no generarían una complejidad mayor al procedimiento.

$$
\begin{aligned}
& \text { Malla } 1^{\prime}=\left[\begin{array}{lllllll}
\mathrm{s} 4 & \mathrm{~s} 5 & \mathrm{~s} 13 & \mathrm{~s} 14 & \mathrm{~s} 15 & \mathrm{~s} 16 & \mathrm{~s} 17
\end{array}\right] \\
& \text { Malla } 2^{\prime}=\left[\begin{array}{llllll}
\mathrm{s} 6 & \mathrm{~s} 7 & \mathrm{~s} 11 & \mathrm{~s} 12 & \mathrm{~s} 13 & \mathrm{~s} 14
\end{array}\right]
\end{aligned}
$$

Con la nueva estructura (Figura 4) es más sencilla la búsqueda de la nueva topología radial que permite la restauración del sistema bajo los objetivos definidos. Lo anterior conlleva a que el proceso de exploración del universo de posibles soluciones sea realizado de manera más eficiente, lo que implica menores tiempos de simulación con mejores resultados.

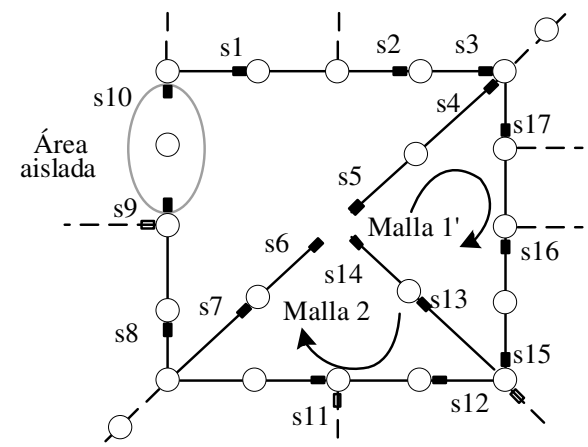

Figura 4. Condición del nuevo sistema.

\section{ALGORITMO DE RESTAURACIÓN DESARROLLADO}

El algoritmo desarrollado considera la minimización multiobjetivo de los índices de pérdidas (1) y número de maniobras (2), considerando las restricciones de radialidad, rango de tensión (3) y límites de corrientes (4).

El algoritmo cuenta con varias etapas que en su conjunto permiten encontrar un grupo de topologías radiales que permiten restablecer el servicio.

Para que el algoritmo esté preparado para enfrentar un proceso de restauración, es necesario que la data de la red se encuentre cargada en el sistema. Esto permite conocer sus parámetros, elementos de maniobra, determinar las mallas fundamentales del sistema original y ajustar los parámetros del NSGA-II. Cuando una falla ocurre, ésta 
debe ser localizada en el sistema. Con esta información y la condición de prefalla, el algoritmo identifica los elementos de maniobra que permiten aislar la o las fallas presentes.

Luego, modifica los vectores de mallas fundamentales según el punto de localización de la falla. Posteriormente, aplicando el NSGA-II, la codificación real y los operadores genéticos especializados, el algoritmo escogerá un conjunto reducido de soluciones eficientes que minimizan ambos objetivos y además cumplen las restricciones establecidas. Todo esto se resume en el diagrama de la Figura 5.

Para evaluar el objetivo asociado a las pérdidas, las restricciones de tensión y de corriente, se utilizará un flujo de carga para sistemas radiales (suma de potencias [20]) lo que permite una evaluación rápida de los individuos dentro del proceso evolutivo.

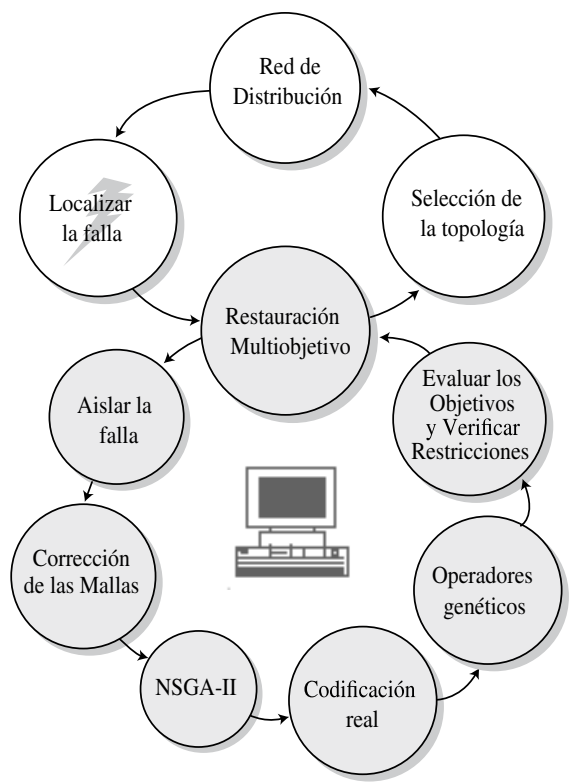

Figura 5. Diagrama del Algoritmo de restauración desarrollado.

\section{APLICACIONES Y ANÁLISIS DE RESULTADOS}

En esta sección el algoritmo será probado en 2 sistemas. El primero de ellos fue obtenido de la referencia [21] y es conocido como sistema Baran, Figura 6. El segundo es un sistema de distribución real de nuestro país de $15 \mathrm{kV}$ que tiene 174 líneas y 163 barras, el cual se muestra en la Figura 9.

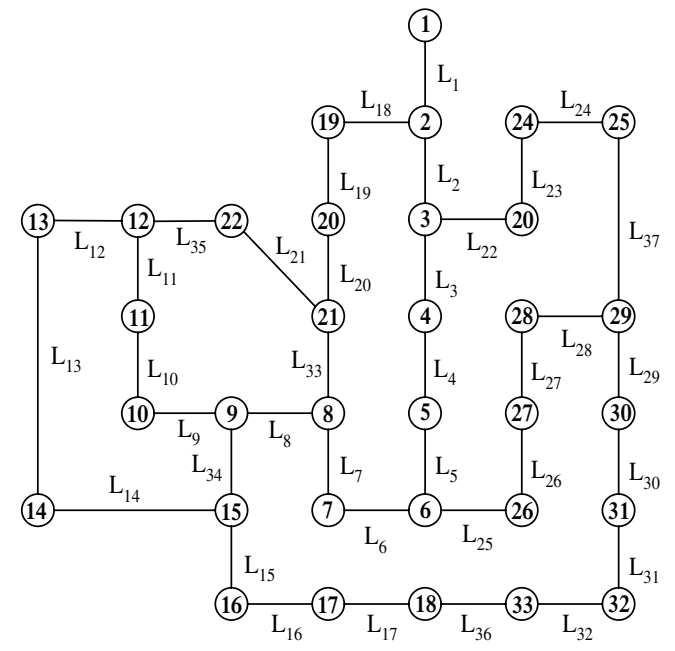

Figura 6. Sistema Baran.

\section{Caso 1: Sistema Baran con falla en línea 3.}

En el caso 1 se simuló el sistema Baran considerando una falla en la línea número 3. A modo de contabilizar el número de maniobras se utilizó como condición inicial del problema la configuración que minimiza las pérdidas, es decir, las líneas [7 91432 37] abiertas [17].

Esta configuración tiene un nivel de pérdidas de 139 kW.

Los resultados mostrados en la Tabla 1 fueron obtenidos considerando una población de 15 individuos y 30 generaciones.

El tiempo total de simulación fue de 7 segundos evaluando 480 individuos valor que corresponde solo al $7 \%$ del universo de posibles soluciones.

En la Figura 7 se muestran los resultados de la Tabla 1, donde con un punto se muestra la evaluación de un pequeño universo de candidatos y con triángulos se muestra la frontera de Pareto encontrada para este problema.

Tabla 1. Resultados Sistema Baran, falla en línea 3.

\begin{tabular}{|c|c|c|c|}
\hline & $\boldsymbol{P}[\mathbf{k W}]$ & $\boldsymbol{N M}$ & Topologías \\
\hline 1 & 165.1673 & 11 & $36-10-34-27$ \\
\hline 2 & 180.4528 & 3 & $32-9-14-7$ \\
\hline 3 & 165.6209 & 5 & $32-9-14-26$ \\
\hline 4 & 165.3241 & 9 & $36-9-34-27$ \\
\hline 5 & 165.5260 & 7 & $36-9-14-27$ \\
\hline
\end{tabular}




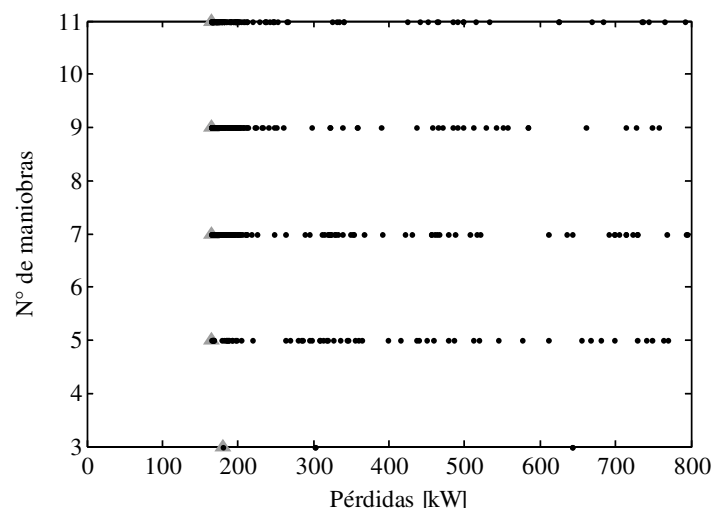

Figura 7. Resultado Sistema Baran, falla línea 3.

\section{Caso 2: Sistema Baran con falla en línea 7.}

En este caso se simuló el sistema Baran con una falla en la línea 7. Se consideró la misma condición inicial que en el caso 1. En este caso no existe región de compromiso entre los objetivos, ya que sólo existe una solución que cumple con la minimización de ambos objetivos, la cual es mostrada en la Tabla 2. Los resultados fueron obtenidos considerando una población de 10 individuos y 20 generaciones. El tiempo total de simulación fue de 2 segundos evaluando 220 individuos, valor que corresponde sólo al 19\% del universo de posibles soluciones.

En la Figura 8 se muestran los resultados de la Tabla 2, donde con un punto se muestra la evaluación de un pequeño universo de candidatos y con un triángulo se muestra la solución eficiente del problema.

Tabla 2. Resultado Sistema Baran, falla en línea 7.

\begin{tabular}{|c|c|c|c|}
\hline & $\mathbf{P}[\mathbf{k W}]$ & NM & Topología \\
\hline 1 & 136.5158 & 3 & $37-14-32$ \\
\hline
\end{tabular}

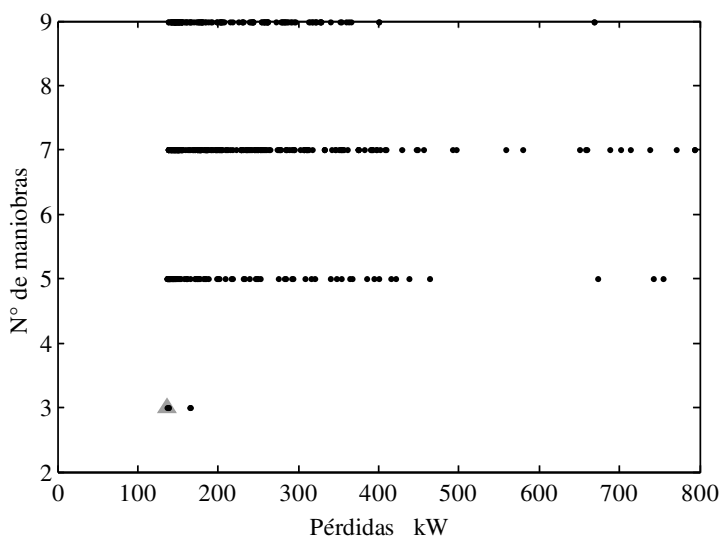

Figura 8. Resultado Sistema Baran, falla línea 7.
Caso 3: Sistema Real con falla en línea 134.

En este caso se simuló el sistema Real con una falla en la línea 134. A modo de contabilizar el número de maniobras se utilizó como condición inicial del problema la configuración que minimiza las pérdidas, es decir, las líneas [6 16870166165164 99], abiertas. Los resultados mostrados en la Tabla 3 fueron obtenidos considerando una población de 10 individuos y 100 generaciones. El tiempo total de simulación fue de 22 segundos evaluando 1.020 candidatos.

En la Figura 10 se muestran los resultados de la Tabla 3, donde con un punto se muestra un pequeño universo de posibles soluciones y con triángulos se muestra la frontera de Pareto encontrada para este problema. El algoritmo además entrega como resultado las líneas que son necesarias desconectar para aislar la falla, las que en este caso corresponden a 132 y 136 . Además, indica las cargas que producto de la falla se mantendrán sin suministro. En este caso las cargas sin suministro serán las asociadas a las barras que utilizan las líneas 133, 134 y 135.

Tabla 3. Resultado Sistema Real, falla en línea 134.

\begin{tabular}{|c|c|c|c|}
\hline & $\mathbf{P}[\mathbf{k W}]$ & NM & Topologías \\
\hline 1 & 195.2 & 7 & $7-168-70-166-165-101$ \\
\hline 2 & 200.6 & 3 & $6-68-70-166-165-99$ \\
\hline 3 & 196.6 & 5 & $6-168-70-166-165-101$ \\
\hline
\end{tabular}

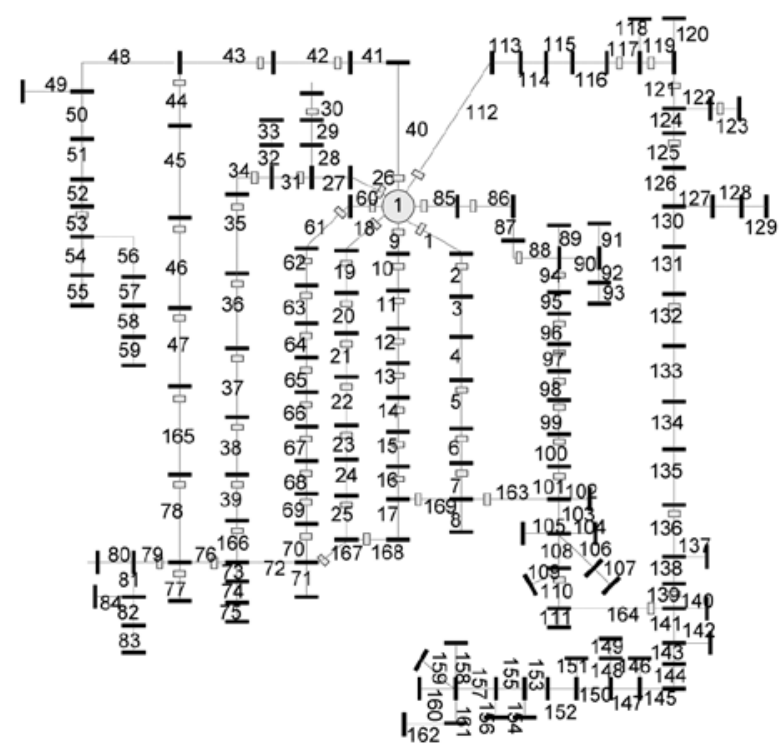

Figura 9. Sistema Real. 


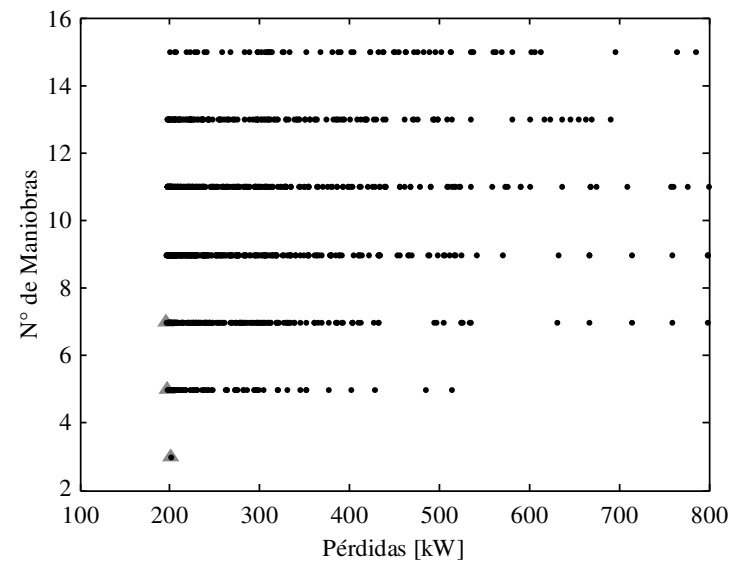

Figura 10. Resultado Sistema Real, falla línea 134.

\section{Caso 4: Sistema Real con falla simultánea en las líneas 4 y 45.}

En este caso se simuló el sistema Real con una falla simultánea en las líneas 4 y 45 . Se consideró la misma condición inicial que en el caso 3. Los resultados mostrados en la Tabla 4 fueron obtenidos considerando una población de 10 individuos y 100 generaciones. El tiempo total de simulación fue de 23 segundos evaluando 1.020. En la Figura 11 se muestran los resultados de la Tabla 4, donde con un punto se muestra un pequeño universo de posibles soluciones y con triángulos se muestra la frontera de Pareto encontrada para este problema. El algoritmo además entrega como resultado las líneas que son necesarias desconectar para aislar la falla, las que en este caso corresponden a $2,5,44$ y 46 . Además indica las cargas que producto de la falla se mantendrán sin suministro. En este caso las cargas sin suministro serán las asociadas a las barras que utilizan las líneas 2, 3, 4, 44 y 45.

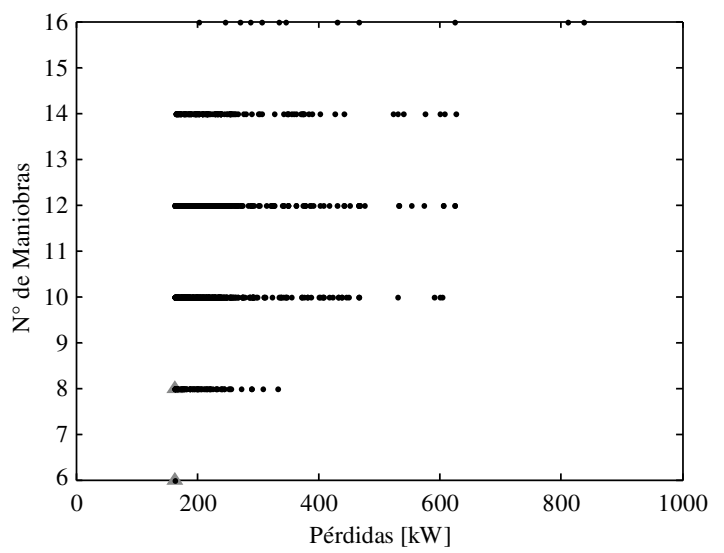

Figura 11. Resultado Sistema Real, falla simultánea en líneas 4 y 45.
Tabla 4. Resultado Sistema Real, falla simultánea en líneas 4 y 45.

\begin{tabular}{|c|c|c|c|}
\hline & $\mathbf{P}[\mathbf{k W}]$ & NM & Topologías \\
\hline 1 & 162.3 & 8 & $168-70-166-164-100$ \\
\hline 2 & 162.3 & 6 & $168-70-166-164-99$ \\
\hline
\end{tabular}

\section{CONCLUSIONES}

En este artículo se desarrolla un modelo de optimización multiobjetivo que aborda el problema de la restauración del servicio eléctrico para las redes de distribución de media tensión.

El modelo desarrollado utiliza una técnica evolutiva de optimización multiobjetivo, denominada NSGA-II, que basa su búsqueda de las soluciones de compromiso utilizando los conceptos de eficiencia de Pareto.

El modelo propone utilizar las pérdidas y el número de maniobras como funciones a optimizar en este problema. El número de maniobras se debe a que se reconoce en este indicador la importancia de minimizar los tiempos de restauración, lo cual se encuentra asociado a los índices de confiabilidad. Por otra parte, se utilizan las pérdidas, ya que representa el indicador asociado a los costos de la distribución. No obstante lo anterior, la naturaleza del modelo desarrollado permite incluir o considerar otros indicadores a ser optimizados.

La comparación de los resultados del algoritmo de prueba implementado, con la frontera de puntos eficientes encontrada a través de una búsqueda exhaustiva, demuestra la eficacia y eficiencia del modelo desarrollado, en la búsqueda del conjunto solución. Lo anterior se debe a las bondades del NSGA II para dar solución a problemas complejos y a la estrategia de codificación y operadores genéticos utilizados.

Aun cuando el algoritmo de prueba fue implementado en ambiente MATLAB, los tiempos de simulación fueron menores a un minuto, lo cual se ajusta a los requerimientos de un algoritmo que debe entregar resultados bajo un estado de contingencia.

Finalmente, el modelo desarrollado tiene la particularidad de abordar problemas de restauración de redes eléctricas que presenten la condición de múltiples fallas simultáneas, simplificando la operación del algoritmo. 


\section{AGRADECIMIENTOS}

Los autores de este trabajo agradecen el apoyo del Fondo Nacional de Desarrollo Científico y Tecnológico FONDECYT a través del proyecto $\mathrm{N}^{\circ} 11070019$ y a la Dirección de Investigación de la Pontificia Universidad Católica de Valparaíso proyecto N $^{\circ} 037.117 / 2008$.

\section{REFERENCIAS}

[1] J. Witte, S. Mendis, M. Bishop and J. Kischefsky. "Computer - aided recloser applications for distribution systems". IEEE Computer application in power system. Vol. 5 Issue 3, pp. 27-32. July 1992.

[2] M.M. Adibi. "Power system restoration". IEEE Press. First Edition. New York, USA. 2000.

[3] R. Brown and A. Hanson. "Impact of two-stage service restoration on distribution reliability". IEEE Transaction on Power System. Vol. $16 \mathrm{~N}^{\circ} 4$, pp. 624-629. November 2001.

[4] C. Chen, C. Lin and H. Tsai. "A rule-based expert system with colored petri net models for distribution system service restoration". IEEE Transaction on Power System. Vol. 17 No 4, pp. 1073-1080. November 2002.

[5] M. Tasai. "Development of an object-oriented service restoration expert system with load variations". IEEE Transaction on Power System. Vol. $23 \mathrm{~N}^{\mathrm{o}} 1$, pp. 219-225. February 2008.

[6] C. Huang. "Multiobjective service restoration of distribution of distribution systems using fuzzy cause-effect networks". IEEE Transaction on Power System. Vol. 18 No 2, pp. 867-874. May 2003.

[7] D. Popovic and Z. Popovic. "A risk management procedure for supply restoration in distribution networks". IEEE Transaction on Power System. Vol. $19 \mathrm{~N}^{\circ}$ 1, pp. 221-228. February 2004.

[8] Y. Ke. "Distribution feeder reconfiguration for load balancing and service restoration by using G-nets inference mechanism". IEEE Transaction on Power Delivery. Vol. 19 No 3, pp. 1426-1433. July 2004.

[9] W. Li, P. Wang, Z. Lim and Y. Liu. "Reliability evaluation of complex radial distribution systems considering restoration sequence and network constraints". IEEE Transaction on Power Delivery. Vol. $19 \mathrm{~N}^{\circ}$ 2, pp. 753-758. April 2004.

[10] S. Toune, H. Fudo, T. Genji, Y. Fukuyama and Y. Nakanishi. "Comparative study of modern heuristic algorithms to service restoration in distribution systems". IEEE Transaction on Power Delivery. Vol. $17 \mathrm{~N}^{\circ}$ 1, pp. 173-181. January 2002.

[11] D. Shin, J. Kim, T. Kim, J. Choo and C. Singh. "Optimal service restoration and reconfiguration of network using Genetic-Tabu algorithm". Electric Power Systems Research. Vol. 71, pp. 145-152. January 2004.

[12] W. Chen, M. Tsai and H. Kuo. "Distribution system restoration using the hybrid Fuzzy-Grey method". IEEE Transactions on Power Systems. Vol. $20 \mathrm{~N}^{\mathrm{o}}$ 1, pp. 199-2005. February 2005.

[13] A. Augugliaro, L. Dusonchet and E. Riva "Envolving non-dominated solutions in multiobjective service restoration for automated distribution networks". Electric Power System Research. Vol. 59, pp. 185195. July 2001.

[14] Y. Kumar, B. Das and J. Sharma. "Multiojective, multiconstraint service restoration of electric power distribution system with priority customers". IEEE Transaction on Power Delivery. Vol. $23 \mathrm{~N}^{\circ} 1$. January 2008.

[15] J. Mendoza, L. Villaleiva, M. Castro and E. Lopez. "Evaluación de Algoritmos Evolutivos Multiobjetivos para la Toma de Decisiones en el Problema de Reconfiguración de Redes Eléctricas de Media Tensión”. XVIII Congreso de la Asociación Chilena de Control Automático ACCA. Santiago, Chile. 2008.

[16] K. Deb, A. Pratap, S. Agarwal and T. Meyarivan. "A fast and elitist multiobjective genetic algorithm: NSGA-II". IEEE Transaction Evolutionary Computing. Vol. $6 \mathrm{~N}^{\circ} 2$, pp. 182-197. April 2002.

[17] J. Mendoza, R. López, D. Morales, E. López, $\mathrm{Ph}$. Dessante and R. Moraga. "Minimal Loss Reconfiguration Using Genetic Algorithms with Restricted Population and Addressed Operators: Real Application". IEEE Transactions on Power Systems. Vol. 21 N $^{\circ}$ 2, pp. 59-67. May 2006. 
[18] B. Enacheanu, B. Raison, R. Caire, O. Devaux, W. Bienia and N. HadjSaid. "Radial Network Reconfiguration Using Generic Algorithm Based on the Matroid Theory". IEEE Transactions on Power System. Vol. $23 \mathrm{~N}^{\mathrm{o}}$ 1, pp. 186-195. February 2008.

[19] J. Mendoza, M. López and E. López. "Multiobjective Reconfiguration Considering Power Losses and Reliability Index for Distribution Network". Congreso IEEE ANDESCON. Cusco, Perú. 2008.
[20] A. Khan, R. Broadwater, and A. Chandrasekaran, "A Comparative Study of Three Radial Power Flow Methods". Proceedings of the IASTED International Symposium, High Technology in the Power Industry, Arizona, pp. 262-265. March 1988.

[21] M.E. Baran and F.F. Wu. "Network Reconfiguration in Distribution System for Loss Reduction and Load Balancing”. IEEE Trans. On PWRD. Vol. 4 $\mathrm{N}^{\circ}$ 2, pp. 1401-1407. 1989. 